\title{
MULTISITE ADSORPTION AND ORDER-DISORDER TRANSITIONS IN ONE- AND TWO-DIMENSIONAL PHASES CONDENSED ON FOREIGN SUBSTRATE
}

\section{B. MUTAFTSCHIEV and A. BONISSENT}

Centre de Recherche des Mécanismes de la Croissance Cristalline, Faculté des Sciences de St-Jérôme, 13397 Marseille Cedex 4, France

\begin{abstract}
Résumé. - On calcule pour des modèles unidimensionnels les isothermes et les variations d'énergie libre dans le cas d'adsorption multisite « un pour $n$ » (c'est-à-dire l'occupation moyenne de $n$ sites d'adsorption par une molécule adsorbée). On montre qu'aucune transition du premier ordre ne peut être attendue entre une phase désordonnée condensée (un pour $n$ ) et la phase solide ordonnée (un pour un).

L'entropie de configuration d'une phase adsorbée bidimensionnelle un pour $\boldsymbol{n}$ est calculée numériquement par une simulation de remplissage séquentielle au hasard. Dans ce cas, la phase bidimensionnelle est construite d'une manière désordonnée et le système peut basculer d'un état désordonné à un état ordonné.
\end{abstract}

\begin{abstract}
Isotherms and free energy variations at equilibrium in the case of fixed one-to-n adsorption (i.e. the average occupation of $n$ adsorption sites by one adsorbed molecule) are calculated for one-dimensional models and their properties are discussed. It proceeds that no first order transition can be expected between a condensed (one-to- $n$ ) disordered phase and the ordered (one-to-one) solid phase. Configurational entropy of an adsorbed two-dimensional one-to- $\boldsymbol{n}$ phase is computed numerically during a random sequential filling simulation. Since in this case, the two-dimensional phase is built in a disordered manner, the system can swing abruptly from disordered to ordered state.
\end{abstract}

1. Introduction. - The experimental works of Thomy, Duval, Régnier et al. [1-5] show that adsorbed layers of rare gases and simple hydrocarbons on lamellar substrates undergo numerous phase transitions, the thermodynamic parameters of which are easily measurable. For this reason, and according to the idea that the structure of the two-dimensional (2 D) condensed phases should be simpler than that of the three-dimensional (3 D) phases, one is tempted to consider 2 D-phases as good models for studying the phase transitions in general. So, one defines [3] not only a 2 D-gas and $2 \mathrm{D}$-solids, but also a $2 \mathrm{D}$-liquid, having typically a lower density with respect to the solids and a great mobility. Unfortunately, while the liquid behaviour of this phase, whose domain of existence is well known, has not yet been directly proved (e.g. by a structure determination technique) we know almost nothing about the thermodynamic stability of the multitude of $2 \mathrm{D}$-ordered phases (some of them with rather low density) observed by low energy electron diffraction in other systems. Thus, the $2 \mathrm{D}$-liquid in the particular case of adsorption of rare gases on lamellar substrates is still highly hypothetical, the more because two points call in question its existence. a) 2 D-layers can be realised on a foreign substrate only if they adhere on it better than on their own substrate (that is the 3 D-crystal of the same substance). Even a weak energetic surface structure of the foreign substrate should then be opposed to the existence of a $2 \mathrm{D}$-liquid layer, in the same way as crystals are mostly resisting surface melting at temperatures lower than the melting point. As regards the lamellar substrates, in particular graphite, the different authors hesitate between two possibilities for the depth of the potential wells on the basal face. A negligible depth $[6,7,8]$ might justify the existence of a 2 D-liquid layer, while large depth could explain [4] the rearrangement and lateral compression of the solid layers, leading to registry.

$b$ ) In the case of a perfectly smooth substrate (from energetic point of view), the key word high mobility is not sufficient to justify the existence of a phase, in a whole range of thermodynamic conditions, if its individuality is not asserted by a particular structure. It is known that $3 \mathrm{D}$-liquids are characterized by a structure, resulting from short range order (in the case of monoatomic liquids, the first coordination shell of an atom shows statistically a five fold symmetry $[9,10])$ and long range 
disorder. At the melting point the structure of the liquid is just as stable as that of the crystal, although the latter is completely different (in the case of monoatomic crystals, the first coordination shell of an atom shows six-fold symmetry). The increased mobility of a liquid comes from its structure inspite of the trivial fact that the mean kinetic energy of the molecules in the crystal and in the liquid at the melting point is the same.

Nothing similar occurs in the case of a 2 D-liquid. A simple hard sphere model shows clearly enough that both compact and nearly compact phases have exactly (or statistically) the same unique six-fold symmetry in the first coordination shell ( $\left.{ }^{1}\right)$. This is one of the reasons why the ordering at increasing density of a disordered 2D-phase is likely to occur continuously as will be shown in the part 3 of this paper.

When searching for the possible disordered two-dimensional arrangements of spherical molecules by a model representing the adsorption of Krypton or Xenon on the basal plane of graphite, one notices a fact that seems to have been overlooked.

It is well known that the theory of the multiple layer adsorption and condensation, worked out by Halsey et al. [14, 15], and inspired by the Frumkin [16]-Fowler [17] isotherm, is well verified in the case of rare gas adsorption on graphite [3]. This is quite astonishing in view of the rough hypotheses on which the Frumkin-Fowler isotherm is based, namely :

(i) a fixed adsorption, that is the mean stay time of a molecule in an adsorption site uncomparetely larger than the time of transfer from one site to another,

(ii) an independence of the configurational entropy from the potential energy ; the entropy is calculated from the unweighted number of all possible configurations of the system (BraggWilliams approximation [18]).

Such a treatment should not be restrictive as regards the number of sites covered by a molecule during its adsorption in one of them, i.e. the ratio $n$ between the number $N_{\mathrm{s}}$ of all adsorption sites and that $\left(N_{a, \max }\right)$ of the adsorbed molecules in a compact monolayer :

$$
n=N_{\mathrm{s}} / N_{\mathrm{a}, \max } .
$$

This number is always tacitly assumed to be unity, even though one knows that in the case of adsorption of $\mathrm{Kr}$ and $\mathrm{Xe}$ on graphite it is equal to

( ${ }^{t}$ Structural studies on hard sphere random models of simple liquids with the help of Voronoi polyhedra show that whereas in a $3 \mathrm{D}$-liquid there is statistically a difference between the number of faces of the polyhedra for a crystal (12) and for a liquid $(\simeq 14)[11,12]$, the Voronoi polygons in a two-dimensionnal space have six sides in both cases of order and disorder [13].
3 [19]. When simple molecules are adsorbed on the (111) face of the f.c.c.-lattice (or the (0001)-face of the h.c.p.-lattice) of a monoatomic isomorph crystal, $n$ is equal to 2 and seems to be the cause of the high configurational entropy of the crystal-melt interface [20].

The reason for always considering $n=1$ is probably the difficulty of calculating, in the other cases, the number of possible configurations of the adsorbed molecules and hence their entropy. We solve the problem only in the case of a one-dimensional model. The properties of the corresponding isotherm will be considered in part 2 of this paper. In part 3 we shall discuss implication of the model as regards the order-disorder transition in a condensed one-dimensional (1D) phase. Some attempts of two-dimensional solutions of the problem will be given in part 4 .

2. The one-dimensional one-to- $n$ model $\left({ }^{2}\right)$. 2.1 ONE- AND TWO-DIMENSIONAL PHASES. - As mentioned, the choice of the one-dimensional model is dictated by mathematical difficulties in treating the two-dimensional one. We can give a good deal of arguments against the eventual doubts regarding the transposition from the first model to the second.

Firstly, the sequels of the simplified Bragg-Williams type entropy calculation (in the oneto-one model) are identical for $1 \mathrm{D}$-, $2 \mathrm{D}$ - and 3 D-phases. Secondly, 1D- and $2 \mathrm{D}$-condensed phases show much more analogies among them than with the $3 \mathrm{D}$-phase, namely :

(i) They both cannot be stable but on a foreign substrate.

(ii) A part of the cohesion throughout the real $1 \mathrm{D}$ and $2 \mathrm{D}$-phases is due to the substrate. This is also influencing the state of the phases, as well as the order of the transitions they undergo. Accordingly, one should be cautious with statements issued from highly idealized or substrate-less $1 \mathrm{D}$ - and 2 D-phases [22, 23, 24].

(iii) As mentioned before, compact or nearly compact 1D- and 2D-phases of simple (e.g. spherical) molecules have only one possible structure against three structures for the $3 \mathrm{D}$-phase.

(iv) From kinetic point of view, a great difference between the $3 \mathrm{D}$-phase and both $1 \mathrm{D}$ - and 2 D-condensed phases is that in the latter case, the

$\left({ }^{2}\right)$ According to equation (1), we shall call one-to-n model the statistical average occupation of $n$ adsorption sites per adsorbed molecule. For the purposes of the configurational calculations it coincides with the real occupation of $n$ sites by one molecule.

The realistic picture, used also in the calculation of the random sequential filling is, however, different [21]. It consists in occupation and partial overlap of more than $n$ sites (depending upon the maximum coordination number). The overlaped sites cannot be occupied by another molecule, but can be overlaped by more than one molecule.

The second picture can be obtained from the first by simple translation of the cellular space by a fraction of the intercellular distance. 
molecules from the vapour have direct access to the bulk of the phases without crossing their surface. This probably insures an instantaneous establishement of equilibrium in most of the systems studied experimentally.

The invoked arguments give us good reasons for studying the properties of an one-dimensional model in order to obtain information concerning a 2 D-phase, in the case of one-to- $n$ adsorption.

2.2 THE BRAGG-WILliamS APPROXIMATION [18]. - It consists in replacing the partition fonction of the adsorbed layer of $N_{\mathrm{a}}$ molecules

$$
\begin{aligned}
Q_{N_{\mathrm{a}}}=g_{1} \exp \left(-U_{1} / k T\right) & +g_{2} \exp \left(-U_{2} / k T\right)+\cdots+ \\
& +g_{l} \exp \left(-U_{l} / k T\right)+\cdots,
\end{aligned}
$$

(where $U_{l}$ is the energy of the $l$-th configuration and $g_{l}$ its number of degeneracy) by :

$$
\begin{array}{r}
Q_{N_{\mathrm{a}}} \simeq\left(g_{1}+g_{2}+\cdots+g_{1}+\cdots\right) \exp (-\bar{U} / k T)= \\
=G \exp (-\bar{U} / k T)
\end{array}
$$

Here $G$ is the total number of configurations that the adsorbed layer can possess and $\bar{U}$ is the average value of the energies $U_{l}$.

The hypothesis of fixed adsorption implies, moreover, that the molecules are vibrating around the adsorption sites and that translation is due to occasional jumps. When a high temperature harmonic approximation is used, (3) can be written as

$$
\begin{aligned}
& Q_{N_{\mathrm{a}}}=G \exp (-\bar{U} / k T) \\
&=G \prod_{i=1}^{3 N_{\mathrm{a}}^{2}}\left(k T / h \nu_{l}\right) \exp \left(\sum_{j=1}^{N_{a}} \phi_{j} / k T\right) \\
&=G\left(2 \pi m k T / h^{2}\right)^{3 N_{\mathrm{l}} / 2} \tilde{v}_{\mathrm{a}}^{N_{a}} \exp \left(N_{\mathrm{a}} \bar{\phi}_{\mathrm{a}} / k T\right) .
\end{aligned}
$$

Here is :

$$
\tilde{v}_{\mathrm{a}}=(k T / 2 \pi m)^{3 / 2}\left(\prod_{l=1}^{3 N_{\mathrm{a}}} \frac{1}{\nu_{l}}\right)^{1 / N_{\mathrm{a}}}
$$

the so-called mean vibrational volume of a molecule in an adsorption site and $\bar{\phi}_{\mathrm{a}}$ is the mean desorption work per molecule.

The variation of the free energy, related to the formation of an adsorption layer of $N_{2}$ molecules from a vapour with a super-(under-)saturation $\Delta \mu=\mu_{\mathrm{g}}-\mu_{\mathrm{g}}^{0}=\mu_{\mathrm{g}}-\mu_{\mathrm{c}}^{0} \quad\left(\mu_{\mathrm{g}}\right.$ and $\mu_{\mathrm{g}}^{0}$ are the chemical potentials per molecule of the super-saturated and the saturated vapor respectively, $\mu_{c}^{0}$ is the chemical potential of the condensed infinite $3 \mathrm{D}$-phase of the same substance at the same temperature) can be written as [25]:

$$
\begin{aligned}
\Delta F_{\mathrm{N}_{\mathrm{a}}}=N_{\mathrm{a}}\left(\phi_{0}-\bar{\phi}_{\mathrm{a}}\right)+N_{\mathrm{a}} k T & \ln \left(\tilde{v}_{\mathrm{o}} / \tilde{v}_{\mathrm{a}}\right)- \\
- & k T \ln G-N_{\mathrm{a}} \Delta \mu,
\end{aligned}
$$

where $\phi_{0}$ and $\tilde{v}_{0}$ are the separation work and the mean vibrational volume of a molecule in the infinite $3 \mathrm{D}$-phase of the adsorbate. Dividing both sides of (5) by $N_{\text {a,max }}$, we have for the reduced free energy variation :

$$
\begin{aligned}
\Delta f=\left(\phi_{0}-\bar{\phi}_{\mathrm{a}}\right)+\theta k T & \ln \left(\tilde{v}_{\mathrm{o}} / \tilde{v}_{\mathrm{a}}\right)- \\
& -\left(1 / N_{\mathrm{a}, \max }\right) \ln G-\theta \Delta \mu,
\end{aligned}
$$

where $0<\theta<1$ is the degree of coverage of the surface. Another current assumption is that the compact adsorption layer is identical (as to structure and coordination) with a lattice plane of the 3 D-infinite crystal of the same substance. If the maximum two-dimensional coordonation number in the first neighbour shell is $z_{1}$ and the first neighbour bond has an energy $\psi_{1}$, the separation work $\phi_{0}$ should be approximately

$$
\phi_{0}=\phi_{0}^{\perp}+z_{1} \psi_{1} / 2
$$

(where $\phi_{0}^{\perp}$ is the separation work of a molecule from its own substrate).

The limitation to first nearest neighbours is not essential for the calculation. We can attribute formally the totality of the interaction energy due to all neighbours to the nearest neighbours only, and write :

$$
\phi_{0}=\phi_{0}^{\perp}+\frac{1}{2} \sum z_{k} \psi_{k}=\phi_{0}^{\perp}+z_{1} \psi / 2
$$

where $z_{k}$ and $\psi_{k}$ are the coordonation number and the bond energy of the $k$-th neighbour, and

$$
\psi=\text { const. }=\sum z_{k} \psi_{k} / z_{1} \text {. }
$$

The mean desorption work $\bar{\phi}_{\mathrm{a}}$ can be written as :

$$
\bar{\phi}_{\mathrm{a}}=\phi_{\mathrm{a}}^{\perp}+\frac{1}{2} \sum z_{i} \psi_{i} W_{i},
$$

where $\phi_{a}^{\perp}$ is the work of desorption from the substrate for $\theta=0$ and $W_{i}$ is the probability for a molecule to have a neighbour in the $i$-th coordonation shell.

In the case of one-to-one model, $W_{i}$ is constant and equal to $\theta$, which allows the use for the mean desorption work of an expression analogous to $\left(6^{\prime}\right)$ :

$$
\bar{\phi}_{\mathrm{a}}=\phi_{\mathrm{a}}^{\perp}+z_{1} \theta \psi / 2
$$

(where $\psi$ has the same value as above). In the case 
of one-to- $n$ adsorption, the more general relation (7) should be used $\left({ }^{3}\right)$.

It must also be remembered in this case that because of the mutual exclusion of a part of the adsorption sites, there are many more coordonation shells in the adsorbed layer than in the compact crystal, i.e.

$$
z_{k} \subset z_{i} \text { and } \psi_{k} \subset \psi_{i}
$$

2.3 LATERAL BOND ENERGIES IN A ONE-TO- $\boldsymbol{n}$ ONE DIMENSIONAL MODEL. - In view of simplicity, we will use for the calculation of bond energies, in the one-to- $n$ adsorption model, the equations $\left(6^{\prime}\right)$ and (7), assuming, however, that the bond energy of lateral interaction is zero for the second nearest neighbour of the compact lattice $(k=2)$. A molecule in the non-compact adsorption layer has then $n$ types of neighbours nearer than the one labeled $k=2$. This assertion is illustrated for the case of one-to-3 adsorption of xenon on graphite (Fig. 1). If one assumes that all hexagons

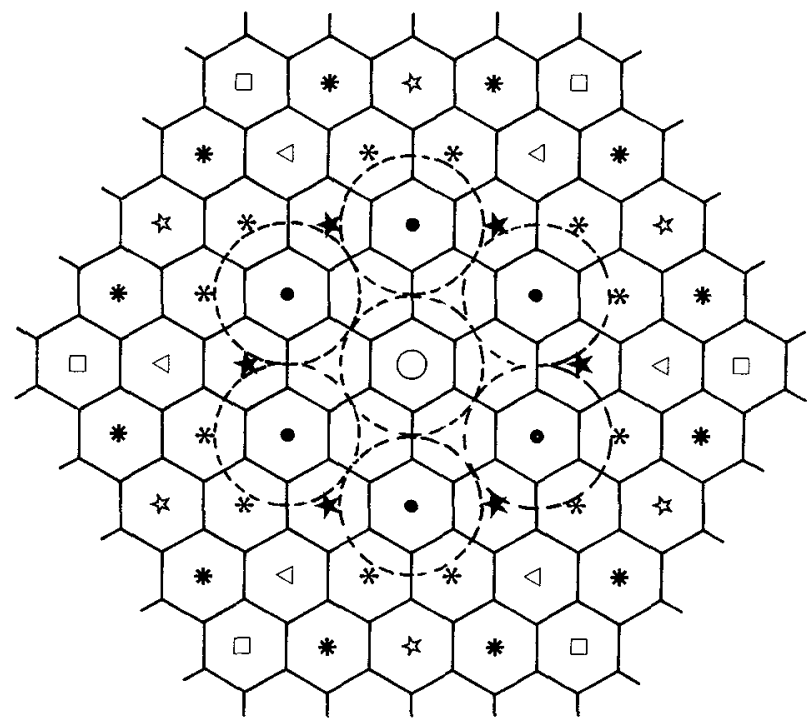

FIG. 1. - Scheme of the sites on the (0001) face of graphite accessible to the adsorption of Krypton (or Xenon) atoms around a central atom $(0)$. The dashed circles represent the ( $\mathrm{Kr}$ or $\mathrm{Xe}$ ) atoms in lattice positions of the compact layer. The symbols of the different types of sites are the same as in table I.

represent potentially identic adsorption sites and that the distance $a$ is exactly equal to the atomic diameter $d$ of the xenon (in fact there is a very small difference between $a=4.26 \AA$ and $d=4.40 \AA$ ), one sees that the central atom o has 6 first nearest neighbours $(k=1)$, at a distance $a$, and 6 second nearest neighbours $(k=2)$, at a distance $\sqrt{3} a$, in the compact two-dimensional lattice, etc. In the non-compact adsorption layer, the nearest

$\left({ }^{3}\right)$ It is still assumed that the compact adsorption layer is isomorph to a lattice plane of the crystal, which is approximately true in the case of krypton and xenon on graphite. neighbours of the atom o can be adsorbed either in the same first neighbour position $(k=1, i=1)$ or in one of the other adsorption sites, which partially exclude themselves mutually. Their distances from the central atom and their maximum coordonation numbers are given in table $I$. Since the bond energy vanishes for $k=2$, it follows that the maximum $i$ to be taken into consideration is equal to $n$.

\section{TABLE I}

Indices, relative distances: $D_{i}=d_{i} / a$ and two-dimensional coordonation numbers. $z_{1}$ of neighbours in the case of adsorption of Krypton (or Xenon) on the basal plane of graphite. The symbols of the sites in the fourth column are the same as in figure 1.

\begin{tabular}{rcrc}
\multicolumn{1}{c}{ Indices } & $D_{i}$ & $z_{\imath}$ & Symbol \\
- & - & - & - \\
$k=1, i=1$ & 1 & 6 & \\
$i=2$ & $2 \sqrt{3 / 3}$ & 6 & $\star$ \\
$i=3$ & $\sqrt{213}$ & 12 & $*$ \\
$k=2, i=4$ & $\sqrt{3}$ & 6 & $\triangle$ \\
$k=3, i=5$ & 2 & 6 & 依 \\
$i=6$ & $\sqrt{393}$ & 12 & $*$ \\
$i=7$ & $4 \sqrt{33}$ & 6 & $\square$
\end{tabular}

The principle of the calculation of the probability for having an $i$-type nearest neighbour in a one-to- $n$ adsorption layer, will be illustrated with the help of a one-to-3 one-dimensional model (Figs 2 and 3).

The probability for a site to be occupied by a molecule (which extends over three sites) is clearly proportional to the degree of coverage. Since there

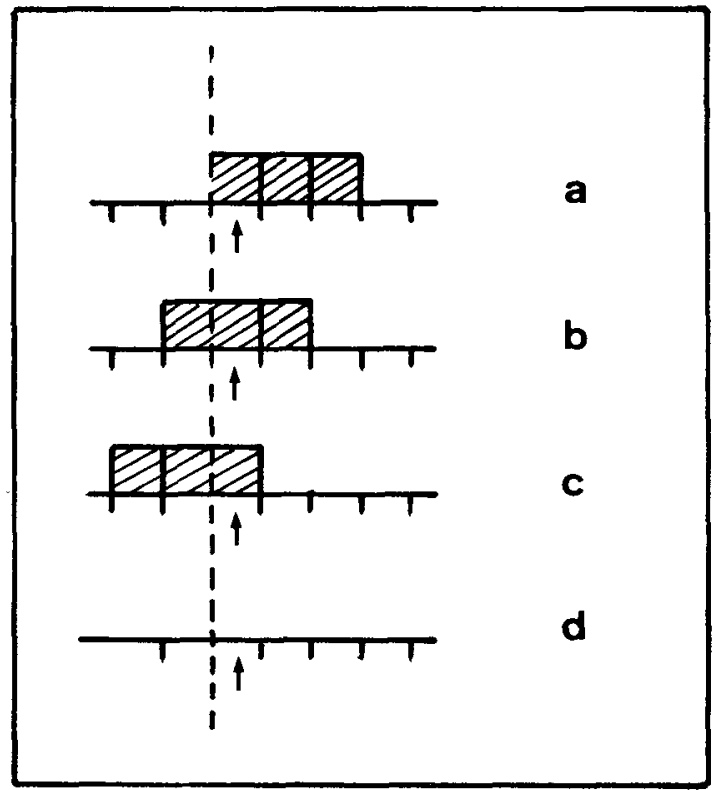

FIG. 2. - States of occupancy of one site $(\uparrow)$ on the surface of a one-dimensional substrate in the case of one-to- 3 adsorption. 


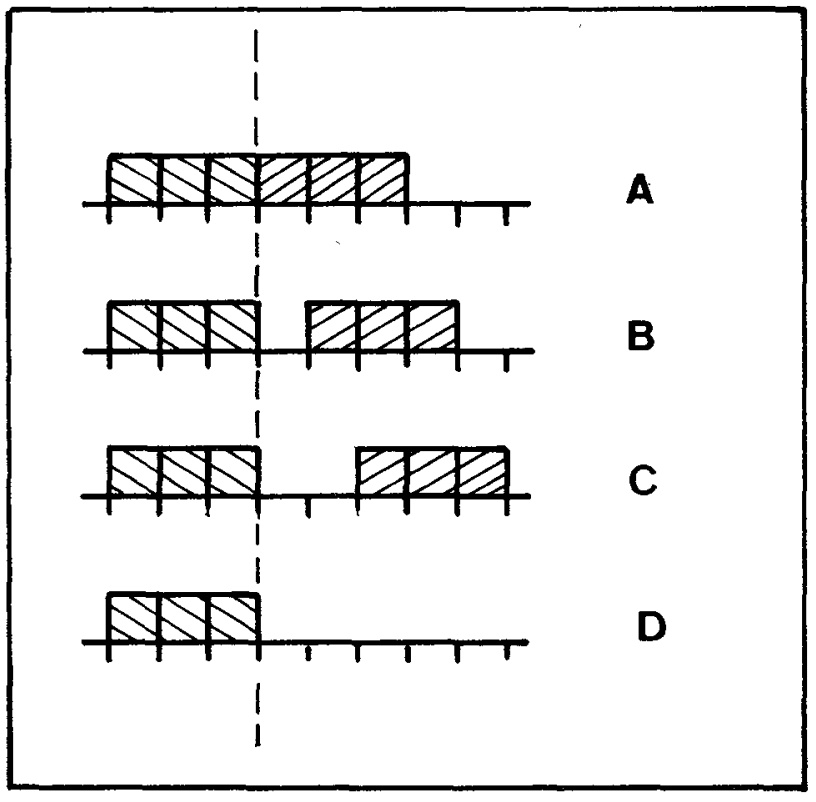

FIG. 3. - Possible unilateral neighbouring of the molecule situated on the left of the dashed line, with bond energies $\psi_{1}(\mathrm{~A})$, $\psi_{2}(\mathrm{~B}), \psi_{3}(\mathrm{C})$ and $0(\mathrm{D})$.

is an equipartition between the three modes of occupation shown in figures $2 a, b, c$, the corresponding probabilities are

$$
w_{\mathrm{a}}^{\prime}=w_{\mathrm{b}}^{\prime}=w_{\mathrm{c}}^{\prime}=k^{\prime} \theta / 3
$$

(where $k^{\prime}$ is a normalization constant). On the other hand, the probability to have the site empty (Fig. 2d) is :

$$
w_{\mathrm{d}}^{\prime}=k^{\prime}(1-\theta) .
$$

(The sum of all four probabilities being unity, $\left.k^{\prime}=1.\right)$

If now one searches for the probabilities of occupation or non occupation of the surface site shown by the arrow, with the suplementary condition [21] that the state of occupation of the site on the left hand side of the dashed line is specified (i.e. it should be empty or filled independently of the site we are considering), the configurations $b$ and $c$ drop. A new normalization gives :

$$
\begin{aligned}
w_{\mathrm{a}}+w_{\mathrm{d}} & =k \theta / 3+k(1-\theta)=1 ; \\
w_{\mathrm{a}} & =\theta /(3-2 \theta) ; \\
w_{\mathrm{d}} & =3(1-\theta) /(3-2 \theta) .
\end{aligned}
$$

For the purposes of the bond energy calculation, the probabilities for occupation of three successive sites neighbouring an adsorbed molecule (Fig. 3) are of interest. They are obtained from the combination of (9) and $\left(9^{\prime}\right)$ and have the values

$$
\begin{aligned}
& W_{\mathrm{A}}=w_{\mathrm{a}}=\theta /(3-2 \theta) ; \\
& W_{\mathrm{B}}=w_{\mathrm{a}} w_{\mathrm{d}}=3 \theta(1-\theta) /(3-2 \theta)^{2} ; \\
& W_{\mathrm{C}}=w_{\mathrm{a}} w_{\mathrm{d}}^{2}=9 \theta(1-\theta)^{2} /(3-2 \theta)^{3} .
\end{aligned}
$$

The substitution of these three probabilities in (7) gives :

$$
\begin{aligned}
\bar{\phi}_{\mathrm{a}}=\phi_{\mathrm{a}}^{\perp}+\left[\psi_{1} \frac{\theta}{3-2 \theta}+3 \psi_{2} \theta\right. & \frac{1-\theta}{(3-2 \theta)^{2}}+ \\
& \left.+9 \psi_{3} \theta \frac{(1-\theta)^{2}}{(3-2 \theta)^{3}}\right],
\end{aligned}
$$

since in this particular case $z_{1}=z_{2}=z_{3}=2$.

In the general case of one-to- $n$ one-dimensional adsorption, we have

$$
\bar{\phi}_{\mathrm{a}}=\phi_{\mathrm{a}}^{\perp}+\theta \sum_{i=1}^{n} \frac{[n(1-\theta)]^{i-1}}{[n(1-\theta)+\theta]^{2}} \psi_{i}
$$

2.4 CONFigurational ENTROPY. - Figure 4 gives an idea of the possible configurations of two linear molecules, each occupying two adsorption sites (one-to 2 -adsorption) of a $3 \times 3$ square lattice. Even for this highly simplified model, the difficulties in the computation of the total number of configurations $(G=44)$, due to steric hindrances, are evident.

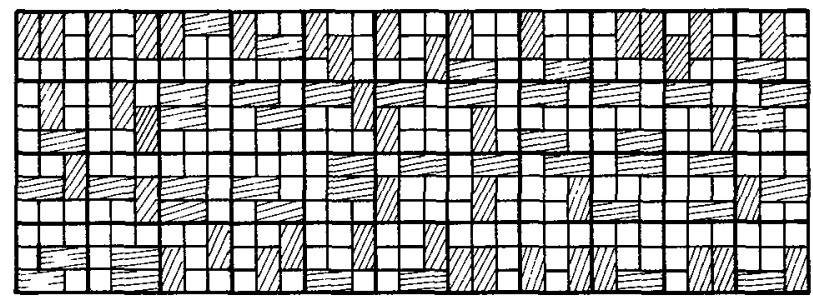

FIG. 4. - Possible configurations of two linear molecules (hatched rectangles), each occupying two adsorption sites of a $3 \times 3$ square lattice.

As mentioned above, at the present stage, we use a one-dimensional model, for which the value of $G$ is easily obtained [26]. If in a linear chain of $N_{\mathrm{s}}$ sites, $n N_{\mathrm{a}}$ sites are occupied by $N_{\mathrm{a}}$ molecules ( $n$-sites each), there are $N_{\mathrm{a}}+N_{\mathrm{s}}-n N_{\mathrm{a}}$ permutable species, $n$-atomic molecules and free sites. $G$ is then directly given by :

$$
G=\left[N_{\mathrm{s}}-(n-1) N_{\mathrm{a}}\right] ! / N_{\mathrm{a}} !\left(N_{\mathrm{s}}-n N_{\mathrm{a}}\right) ! .
$$

The substitution of (6), (11) and (12) into (5') yields after some simplifications (Stirling's approximation) the relation: 


$$
\begin{aligned}
\Delta f= & \theta\left(\phi_{\mathrm{o}}^{\perp}-\phi_{\mathrm{a}}^{\perp}\right)+\psi_{1} \theta\left\{1-\theta \sum_{i=1}^{n} \frac{[n(1-\theta)]^{i-1}}{[n(1-\theta)+\theta]^{i}} \frac{\psi_{i}}{\psi}\right\}+ \\
& +k T\{\theta \ln \theta+n(1-\theta) \ln [n(1-\theta)]-[n(1-\theta)+\theta] \ln [n(1-\theta)+\theta]\}+\theta k T \ln \left(\tilde{v}_{0} / \tilde{v}_{\mathrm{a}}\right)-\theta \Delta \mu,
\end{aligned}
$$

which clearly gives the Fowler isotherm for $n=1$.

If we take as reference state for the supersaturation not more the equilibrium pressure of the infinite 3 D-crystal (at the same temperature), but the equilibrium pressure of the infinite compact 2 D-phase on the same substrate, the new, reduced supersaturation $\Delta \mu^{\prime}$ is defined as [27] :

$$
\Delta \mu^{\prime}=\Delta \mu-\Delta^{*} \mu=\Delta \mu-\left(\phi_{0}^{\perp}-\phi_{\mathrm{a}}^{\perp}\right)-k T \ln \left(\tilde{v}_{\mathrm{o}} / \tilde{v}_{\mathrm{a}, 0}\right),
$$

where $\tilde{v}_{a, 0}$ is the mean vibrational volume per molecule, in the compact layer; $\Delta^{*} \mu$ is the supersaturation at which, according to the idealized (one-to-one) Fowler isotherm, the equilibrium between the condensed (compact) and the diluted 2 D-phases is established.

The substitution of (14) into (13) yields :

$$
\begin{aligned}
\Delta f=\psi_{1} \theta\left\{1-\theta \sum_{i=1}^{n} \frac{[n(1-\theta)]^{i-1}}{[n(1-\theta)+\theta]^{i}} \frac{\psi_{i}}{\psi_{1}}\right\} & + \\
+k T\{\theta \ln \theta+n(1-\theta) \ln [n(l-\theta)]-[n(1-\theta)+\theta] \times & \times \ln [n(1-\theta)+\theta]\}-k T \theta \ln \left(\tilde{v}_{\mathrm{a}} / \tilde{v}_{\mathrm{a}, 0}\right)-\theta \Delta \mu^{\prime} .
\end{aligned}
$$

2.5 PROPERTIES OF THE ONE-TO- $n$ ISOTHERM. - The isotherm for the one-to- $n$ adsorption is obtained from the condition of maximum (negative) variation of (15) at constant temperature :

$$
\begin{aligned}
(\partial \Delta f / \partial \theta)_{T}=\psi_{1}\left\{1-\sum_{i=1}^{n} \frac{\left[n\left(1-\theta_{0}\right)\right]^{\mathrm{k}-1}}{\left[n\left(1-\theta_{0}\right)-\theta_{0}\right]^{2}} \times\left[2 \theta_{0}-\frac{(i-1) \theta_{0}^{2}}{1-\theta_{0}}+\frac{i(n-1) \theta_{0}^{2}}{n(1-\theta)+\theta_{0}}\right] \frac{\psi_{i}}{\psi_{1}}\right\} & + \\
& +k T \ln \frac{\theta_{0}\left[n\left(1-\theta_{0}\right)+\theta_{0}\right]^{n-1}}{\left[n\left(1-\theta_{0}\right)\right]^{n}}-k T \ln \left(\tilde{v}_{\mathrm{a}} / \tilde{v}_{\mathrm{a}, 0}\right)-k T \theta_{0}\left(\partial \ln \tilde{v}_{\mathrm{a}} / \partial \theta\right)_{T}-\Delta \mu^{\prime}=0
\end{aligned}
$$

where $\theta_{0}$ is the equilibrium degree of coverage. The variation of the free energy at equilibrium, $\Delta f_{\text {eq }}$, equal to the variation of the surface free energy of the substrate, multiplied by the area $s=1 / N_{\mathrm{a}, \max }$ of an adsorbed molecule, leads, according to (16) and (15), to :

$$
\begin{aligned}
& \Delta f_{\mathrm{eq}}=\psi_{1} \theta_{0} \sum_{i=1}^{n}\left\{\frac{\left[n\left(1-\theta_{0}\right)\right]^{i-1}}{\left[n\left(1-\theta_{0}\right)+\theta_{0}\right]^{i}}\right. \\
&\left.\times\left[\theta_{0}-\frac{(i-1) \theta_{0}^{2}}{1-\theta_{0}}+\frac{i(n-1) \theta_{0}^{2}}{n(1-\theta)+\theta_{0}}\right] \frac{\psi_{i}}{\psi_{1}}\right\}- \\
& \quad-k T \theta_{0}^{2}\left(\partial \ln \tilde{v}_{\mathrm{a}} / \partial \theta\right)_{T}+k \ln \ln \frac{n\left(1-\theta_{0}\right)}{n\left(1-\theta_{0}\right)+\theta_{0}} .
\end{aligned}
$$

In as much as we are unable to calculate the oneto- $n$ isotherm for a two-dimensional model, we hoped that the one-dimensional isotherm (16) would represent better the adsorption of rare gases on graphite $(n=3)$ if the bond energies $\psi_{i}$ in (16) were weighted according to the relation:

$$
\psi_{i}=z_{i} \phi_{i} / 2
$$

where $z_{i}(i=1,2,3)$ is the two-dimensional maximum coordination number of table $I$. The bond energy with the $i$-th neighbour is calculated from the relative distance $D_{i}$ of the same table, according to the Lennard-Jones potential law :

$$
\phi_{i}=\psi_{1}\left[2\left(1 / D_{i}\right)^{6}-\left(1 / D_{i}\right)^{12}\right]
$$

Making the quite current approximation $\tilde{v}_{\mathrm{a}}=\tilde{v}_{\mathrm{a}, 0}=$ const. (whose sequels will be discussed later), the only variable parameter in (16) happens to be $\omega=\psi_{1} / k T$ just as in the case of the Fowler isotherm [17].

In figures 5 and 6 are plotted the isotherms calculated from equations (16) with $n=3$ and $n=1$ (Fowler) for two values of the parameter $\omega$. An important feature is that the increase of $\boldsymbol{n}$ provokes a shift of the step of the isotherm towards lower $\Delta \mu^{\prime}$ and decreases the first order character of the one-dimensional condensation.

The decreasing first order character of the one-dimensional condensation with increasing number of sites per adsorbed molecule is due to the increase of the configurational entropy. One can be 


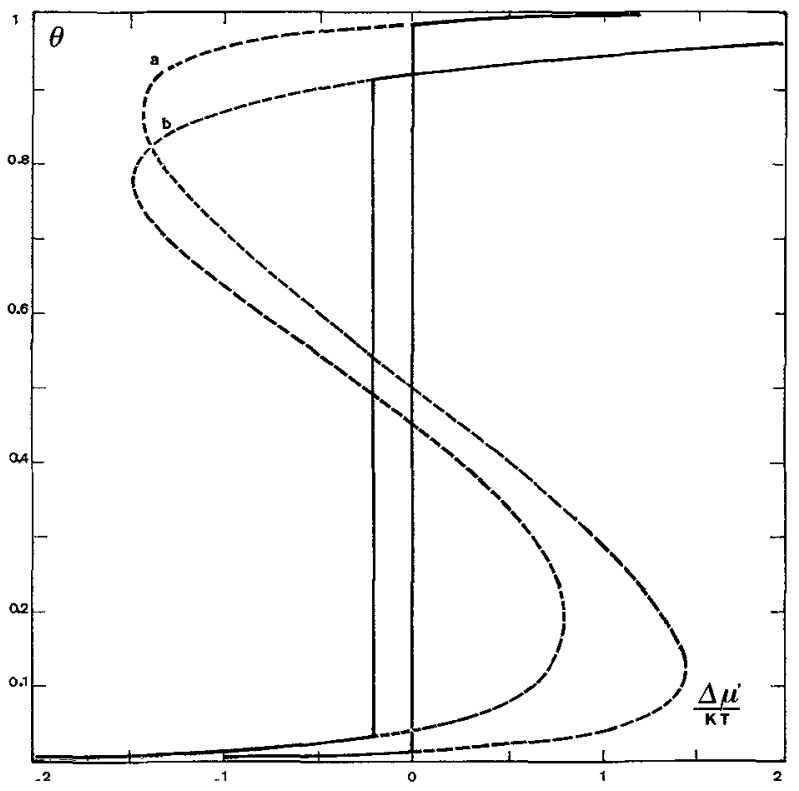

FIG. 5. - Unidimensionnal adsorption isotherms for $\omega=4.5$; curve a : Fowler isotherm $(n=1)$; curve $b$ : one-to- 3 adsorption $(n=3)$. The dashed parts correspond to metastable and unstable states. The positions of the vertical parts are deduced from the $\Delta f / k T$ versus $\Delta \mu^{\prime}$ curves of figure 7 .

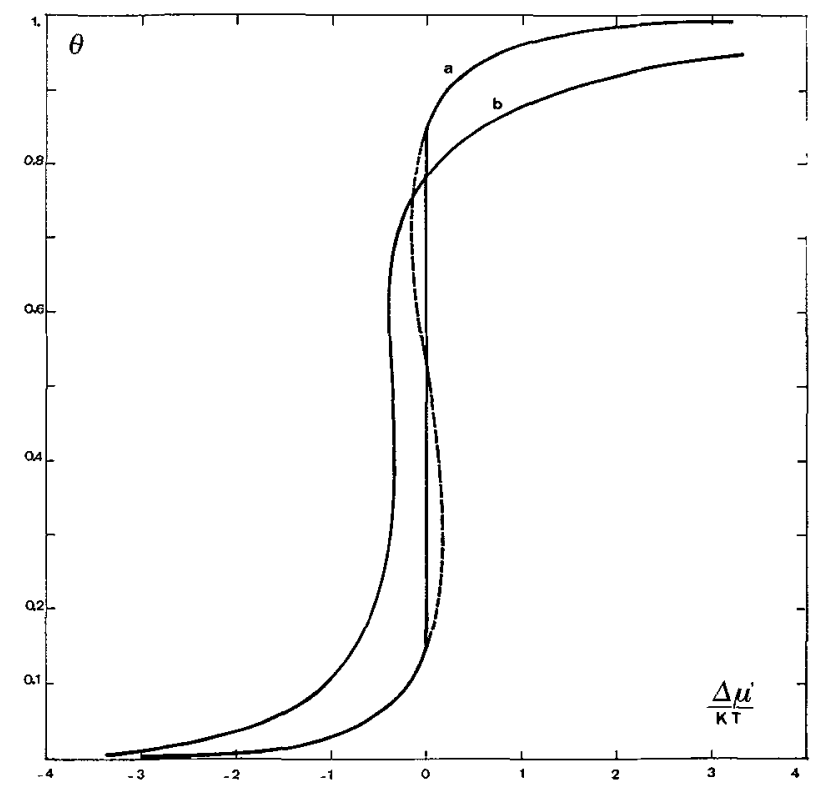

FrG. 6. - Unidimensionnal adsorption isotherms for $\omega=2.5$; curve a : Fowler isotherm $(n=1)$; curve $\mathrm{b}$ : one-to- 3 adsorption $(n=3)$.

easily tempted to let $n$ tend to infinity in order to obtain the configurational entropy of a non-fixed (mobile) layer. This is clearly impossible since the model is valid only for a fixed adsorption, i.e. for the case when the mean vibrational distance $\tilde{a}=\left(\tilde{v}_{\mathrm{a}}\right)^{1 / 3}$ is small compared to the distance between adsorption sites. When vibrational volumes overlap, the entropy should be assimilated to that of a one-dimensional fluid and its calculation becomes problematic. In the considered adsorption of rare gases on graphite, one can be quite sure, however, that this is not the case.

3. Disorder-order transition in the condensed onedimensional layer. - Figure 5 shows that for one and the same supersaturation, the isotherm corresponding to $n=3$ is situated partially above, partially below the Fowler isotherm $(n=1)$. The question which arises immediately is whether or not the system can swing spontaneously (at constant temperature and for a small increase in the supersaturation) from the disordered condensed state, typical for $n=3$ and uncomplete coverage, to the ordered compact state expected from the isotherm for $n=1$.

Figure 7 gives the free energy variation at equilibrium as a function of the supersaturation for the two types of isotherms of figure 5. Since the function $\Delta f_{\text {eq }}\left(\Delta \mu^{\prime}\right)$ can be obtained only from the numerical solution of the equations (16) and (17), curves analogous to those of the figure 7 were computed for a great variety of parameters $\boldsymbol{n}$ and $\psi_{1} / k T$. The result was always the same, namely the free energy curve corresponding to the condensation of a disordered phase $(n>1)$ is situated below the respective curve for condensation of the ordered phase $(n=1)$. Both curves coincide for $\Delta \mu \rightarrow \infty$.

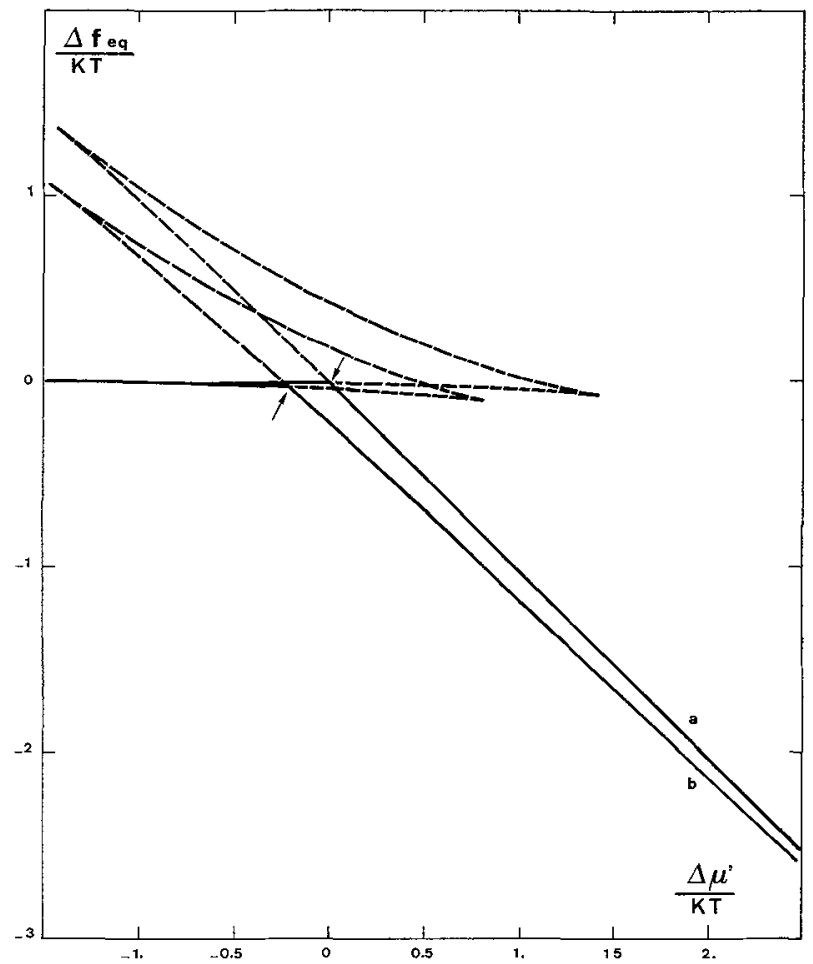

FIG. 7. - Reduced free energy variation at equilibrium, calculated from the isotherms of figure 5; curve a: Fowler adsorption $(n=1)$; curve $\mathrm{b}$ : one-to-3 adsorption $(n=3)$. The dashed parts correspond to metastable and unstable states. The arrows indicate the points of two-phase equilibrium. 
The non crossing of the curves indicates the impossibility, for our model, of first order transition to occur in a condensed one-dimensional state. The importance of this statement is such, that we need to search for eventual flows inherent to the model.

(i) One should remember that the dependence of the mean vibrational volume with the coverage entering both equations (16) and (17) was neglected. This approximation would not greatly affect the isotherm insofar as, $\tilde{v}_{\mathrm{a}}$ being always larger than $\tilde{v}_{\mathrm{a}, 0}$, but tending to it for $\theta_{0} \rightarrow 1$, the two vibrational terms of equation (16) should have opposite signs. It is more hazardous to compare the influence of the vibrational entropy term in equation (17) for the disordered and the ordered condensed layers. However, it is reasonnable to assert that for the same coverage $\theta_{0}$ the (negative) value of the derivate $\left(\partial \ln \tilde{v}_{\mathrm{a}} / \partial \theta\right)_{T}$ will be similar in both cases. The presence in the same term of equation (17) of the square of $\theta_{0}$, should then accentuate (at the same supersaturation) the stability of the phase with lower coverage, i.e. the ordered phase at low supersaturation and the disordered phase at high supersaturation (cf. figure 5). This reinforces the effect obtained by neglecting the vibrational entropy terms.

(ii) Similar arguments can be evoked in answering the questions of what would happen if the disordered condensed phase were much more mobile or were a true fluid. The completely non-localised fluid state implies a further increase of the entropy in respect to that calculated above. The free energy curve corresponding to the liquid state should be shifted to more negative values and no crossing of the curves of figure 7 can be expected.

The impossibility for a first order liquid-solid transition in $1 \mathrm{D}$-(and $2 \mathrm{D}$-) condensed phases to be provoked, by a sole and small variation of the gas pressure seems thus to be strengthened. As forseen in the introduction of this paper, this can be explained by the lack of a break between disordered and ordered structures, contrary to the $3 \mathrm{D}$-phases. The establishement of order throughout the disordered layer by increasing the coverage goes smoothly up to $\theta_{0}=1$. This is favorized kinetically thanks to the accessibility of the bulk to direct exchange with the three dimensional gas.

4. Attemps for solution by two-dimensional one-to$n$ models. - 4.1 RANDOM SEQUENTIAL FILLING AND CONFIGURATIONAL ENTROPY. - The problem of the configurational entropy of a two-dimensional one-to- $\boldsymbol{n}$ adsorbed layer has many common points with the problem of random sequential filling of a two-dimensional cellular space, as can be seen from the following considerations.

The number $G$ of all possible configurations of a system (in our case, that containing $N_{\mathrm{a}}$ molecules which can be placed on $N_{\mathrm{s}}$ sites) is equal to the total number of independant ways for building the system, divided by $N_{\mathrm{a}}$ ! (since each particular configuration can be made up by $N_{\mathrm{a}}$ ! independent manners). A possible numerical calculation of $G$ could consist in letting a computer explore all possible ways for building a system, which is clearly a formidable task. The method we choose, based on the random sequential filling, can be illustrated with the help of the model given in figure 4 .

There are 12 ways of placing the first bi-atomic molecule on the $3 \times 3$ lattice and, according to the placement of that molecule, there are either 6 or 8 ways of placing the second molecule. For 4 possible positions of the first molecule, one has 6 accessible positions for the second one and for 8 possible positions of the first, we have 8 accessible positions for the second. The total number of ways is then $G=(4 \times 6+8 \times 8) / 2 !=44$, as in figure 4 .

If, instead of trying all ways of building the system, we fill randomly the cells, and determine before every filling the mean number of accessible positions of a molecule, we shall state that there are 12 accessible positions before the deposition of the first molecule and in average 7.33 accessible positions for the deposition of the second molecule, hence $G=12 \times 7.33 / 2 !=44$.

The number of configurations of $N_{\mathrm{a}}$ molecules on $N_{1, \mathrm{~s}}$ sites (all accessible for the deposition of the first molecule) is then :

$$
G=\left(N_{\mathrm{t}, \mathrm{s}} \prod_{i=2}^{N_{\mathrm{a}}} \bar{N}_{i, \mathrm{~s}}\right) / N_{\mathrm{a}} !
$$

where $\bar{N}_{i, \mathrm{~s}}$ is the average number of accessible sites before the deposition of the $i$-th molecule.

The calculation of $\bar{N}_{i, s}$, based on the probabilities for random filling of a space, is performed for one-dimensional cellular model only [21, 28, 29].

The logarithm of $G$ in equation (18), proportional to the configurational entropy, was calculated by this method [28]. It is plotted versus the degree of coverage $\theta$ in figure 8 . The model is that of a one-to- 2 occupation. For comparison, in the same figure we plotted the configurational entropy calculated exactly from equation (12).

Figure 8 suggests the following remarks :

(i) The two curves overlap up to degrees of coverage $\theta$ of 0.3 to 0.4 . At higher coverages they diverge strongly and the curve representative of the random filling becomes nil for $\theta=0.8647$. This concentratation limit for a randomly built model, calculated by McQuistan [29] and Widom [28], is confirmed also by computer simulation.

(ii) The difference between the two curves is easily understandable. The values of $\bar{N}_{i, s}$, obtained from configurations with maximum probability, 


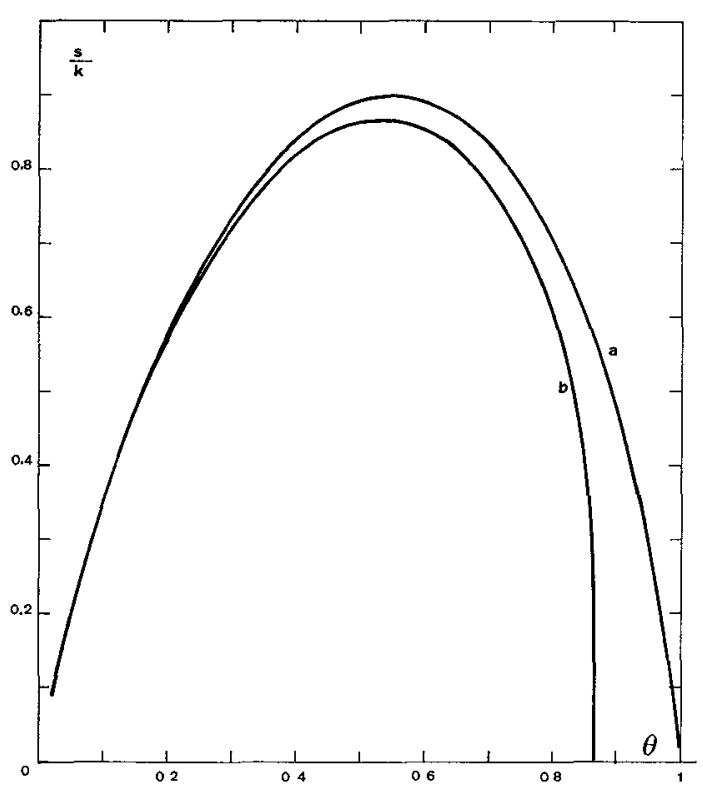

FIG. 8. - Reduced configurational entropy ( $s=k \ln G / N_{\mathrm{a}, \max }$ ), versus degree of coverage, for one-to- 2 adsorption model. Curve $a$ is calculated from equation (12), curve $b$ is issued from the random sequential filling [28].

coincide with the true mean values for small coverages. At high coverages, the number of accessible sites for deposition of a new molecule can be different from zero, only if parts of the system contain very compact and hence ordered $2 \mathrm{D}$-clusters. But, this is almost excluded during the random filling. (iii) Since, the disorder itself is hard to define in a cellular space, it seems to us that the random filling is a fairly acceptable criterion for such a disorder. From this point of view, the (lower) configurational entropy given by the curve $b$ in figure 8 is that of $a$ phase squeezed to remain in a complete disorder, since curve a gives the entropy of all possible states, those of partial or total order included.

\subsection{ISOTHERM AND FREE ENERGY VARIATION OF} A COMPLETELY DISORDERED TWO-DIMENSIONAL PHASE. - Using a random sequential filling algorithm [30], we calculated simultaneously mean potential energies of lateral bonds and configurational entropies of a two-dimensional model representative of the adsorption of Krypton or Xenon on graphite $(n=3$, Lennard-Jones 6/12 pair interaction potential). The dispersion in the average numbers $\bar{N}_{t, \mathrm{~s}}$ of equation (18) calculated from 30 sequential fillings, was always extremely small and the coverage limit $\theta=0.70$ was close to the values obtained by Widom [21] for different models. The free energy variation, determined from the numerical calculation of equation $\left(5^{\prime}\right)$, (with the approximation $\tilde{v}_{\mathrm{a}}=\tilde{v}_{0}$ ) leads to the isotherm $\mathrm{b}$ in figure 9. For comparison the Fowler $(n=1)$ isotherm is also plotted (curve a). One notices that the constrained disorder in the computer built adsorption model provoke a shift of this step to lower supersaturations and the $2 \mathrm{D}$-gas-2 D-liquid transition is overcritical.

In figure 10 are plotted the free energy variations

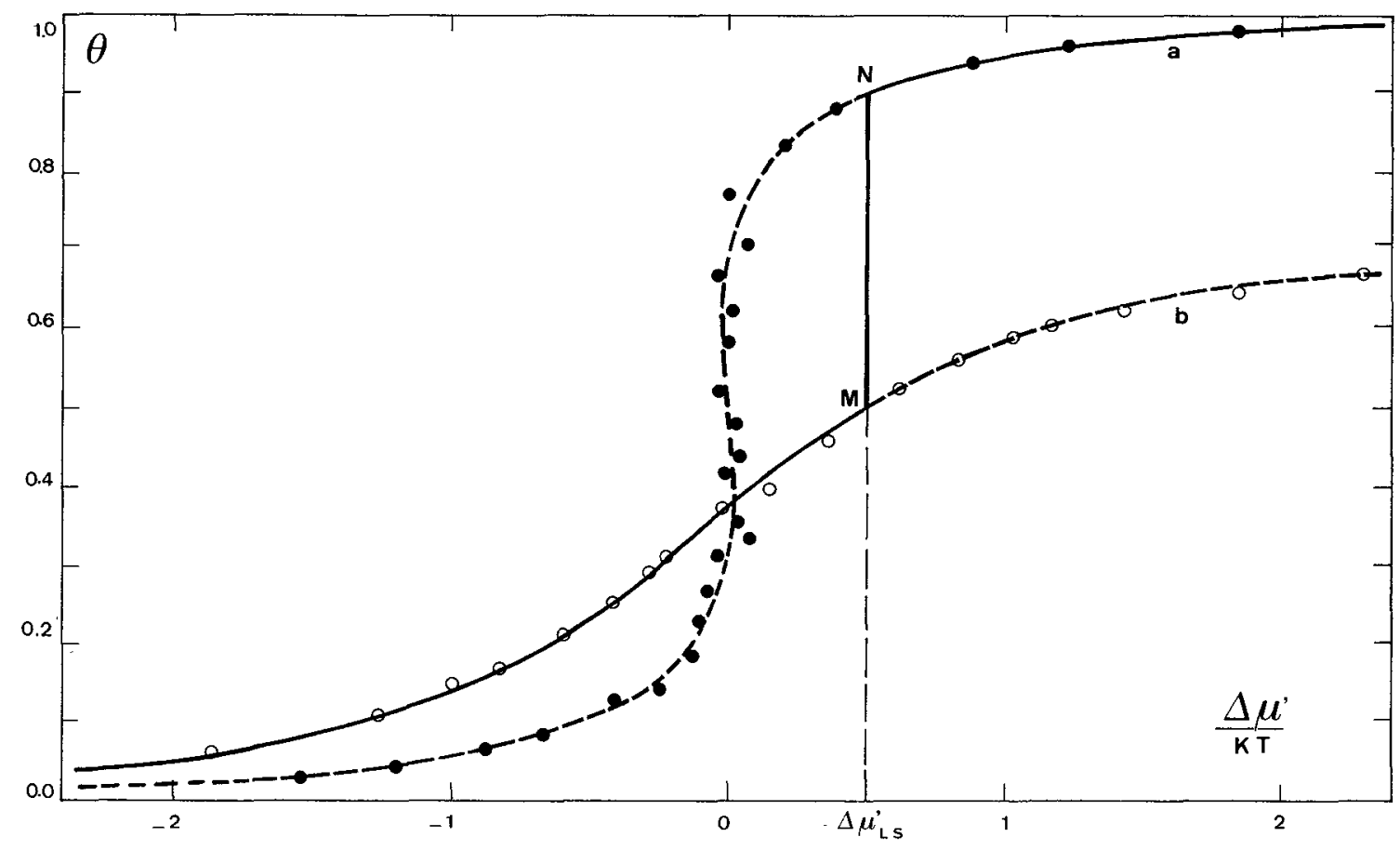

FIG. 9. - Isotherms representative for a two-dimensional adsorption model $(\omega=3.75)$. Both configurational entropy and mean lateral bond energy are average values calculated from the simulation of 30 random sequential fillings. $:$ one-to-one adsorption ( $n=1)$; the curve passing through the points is that calculated exactly from the Fowler isotherm. $\bigcirc:$ one-to-3 adsorption ( $n=3)$. The solid line represents the stable states. $\Delta \mu_{\mathrm{L}}^{\prime}$ is defined in figure 10. 


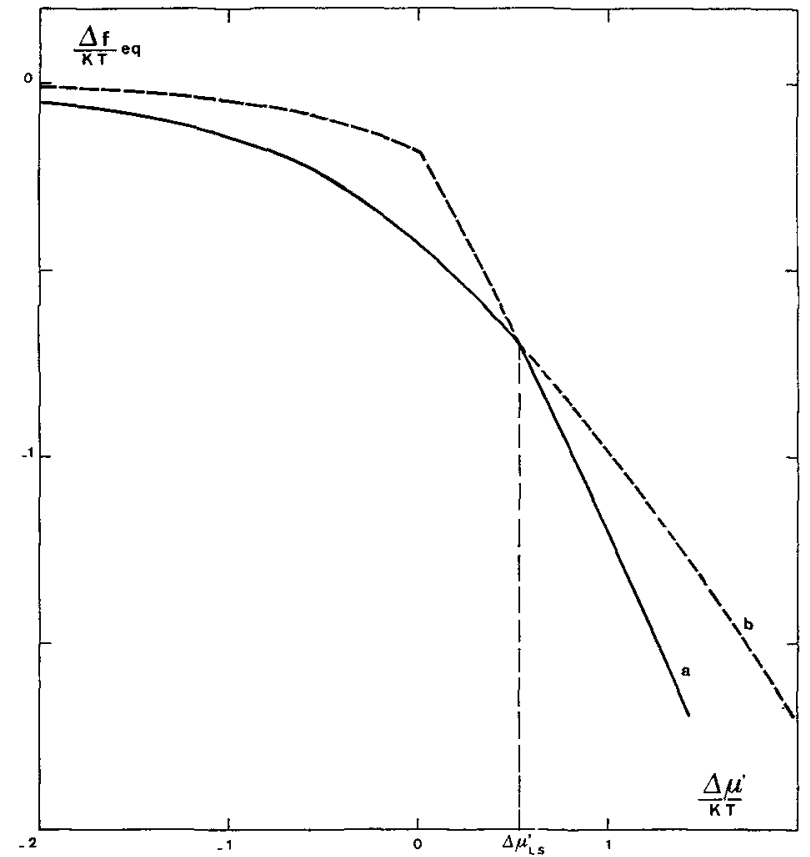

FIG. 10. - Reduced free energy versus supersaturation for the two isotherms of figure 9 ; curve a: one-to-one adsorption curve $b$ : one-to- 3 adsorption. The solid line represents the stable states, $\Delta \mu_{\mathrm{LS}}^{\prime}$ is the equilibrium supersaturation of the order-disorder transition.

at equilibrium $\left(\Delta f_{\mathrm{eq}}\right)$ versus supersaturation, for the two cases represented by the isotherms in figure 9. The discontinuity provoked by the crossing of the two curves is evident. Its consequence is the spontaneous transition inside the condensed disordered layer, at the supersaturation designed as $\Delta \mu_{\mathrm{LS}}^{\prime}$ (Figs 9 and 10), which is different from the supersaturation of the step $\Delta \mu^{\prime}=0$. The coverage passes then suddently from point $M$ to point $N$. The qualitative features of the liquid-solid phase transitions, found experimentally, are thus confirmed.

5. Conclusion. - In spite of the simplicity of the models used in this paper, one fact seems to be established : a discontinuous disorder-order phase transition, at constant temperature, and hence the distinction between fluid and solid, in $1 \mathrm{D}$ - and 2 D-phases, cannot be related simply to a mobility variation, but should result from structural modifications. The split of the first step in the experimental isotherms (if it is not due to a $2 \mathrm{D}$ solid-solid transformation) takes place only in the case of a constrained disordered liquid layer obtained by random sequential filling. The reasons for the eventual existence of such a forced disorder, in a real system, are still not clear.

A possible hypothesis is that before the completion of the first layer $(\theta<1)$ the adsorption in some especially advantageous positions of the second layer (for example holes of the first layer slightly smaller than the dimension of a molecule) can lead temporarily to a lower free energy than the continuous ordering of the first layer. If this is true, the molecules of the $2 \mathrm{D}$-liquid will have nearly the environement of those in the $3 \mathrm{D}$-liquid (i.e. a preferential polytetrahedral packing) [10].

Does this mean that in the future $2 \mathrm{D}-\ll$ liquids » should be spelt $\ll 2 \mathrm{D} »$-liquids ?

References

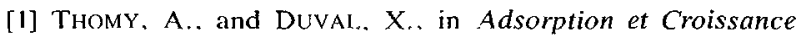
Cristalline, C.N.R.S. Paris (1965) p. 81.

[2] Thomy, A. and Duval, X.. J. Chim. Phys. 66 (1969) 1966.

[3] Thомy, A. and Duval, X.. J. Chim. phys. 67 (1970) 1101.

[4] Thomy, A., Regnier, J., Menaucourt, J. and Duval, X., J. Cryst. Growth 13/14 (1972) 159.

[5] Regnier, J., Ph. D. Thesis, Nancy (1976).

[6] Crowell, A. D. and Young, D. M., Trans. Faraday Soc. 49 (1953) 1080

[7] PACE, E. L., J. Chem. Phys. 27 (1957) 1341.

[8] Price, G. L., Surf. Sci. 46 (1974) 697.

[9] Frank, F. C., Proc. R. Soc. A 215 (1952) 43.

[10] Bernal, J. D., Proc. R. Soc. A 280 (1964) 299.

[11] Bernal, J. D., in Liquids, Elsevier, Amsterdam (1965) p. 25.

112] Scorr, G. D., Nature 188 (1960) 908 ; ibid. 194 (1962) 956.

[13] Finney, J. L., Proc. R. Soc. A 319 (1970) 479.

[14] Champion, W. M. and Halsey, G. D., J. Phys. Chem. 57 (1953) 646.

[15] Singleton, J. H. and Halsey, G. D., J. Phys. Chem. 58 (1954) 1011
[16] Frumkin, A. N., Z. Phys. Chem. 116 (1925) 466.

[17] Fowler, R. and Guggenheim, G. A., Statistical Thermodynamics, Cambridge (1960) p. 466.

[18] Bragg, W. L. and Williams, E. G., Proc. R. Soc. 145 (1934) 699 ; ibid. 151 (1935) 540.

[19] Morrison, J. and Lander, J. J., Surf. Sci. 5 (1966) 163.

[20] SPAEPEN, F., Acta Metall. 23 (1975) 731.

[21] WIDOM, B., J. Chem. Phys. 44 (1966) 3888.

[22] Van Hove, L., Physica 16 (1950) 137.

[23] Pierls, R. E., Ann. Inst. Henri Poincaré 5 (1935) 177.

[24] Kosterlitz, J. M. and Thouless, D. J., J. Phys. C. 6 (1973) 1181 .

[25] Mutaftschiev, B., Surf. Sci. 61 (1976) 93.

[26] MCQuistan, R. B., Nuovo Cimento 58 B (1968) 86.

[27] CASEs, J. M. and Mutaftschiev, B., Surf. Sci. 9 (1968) 57

[28] Widom, B., J. Chem. Phys. 58 (1973) 4043.

[29] MCQuistan, R. B. and Lichrman, D., J. Math. Phys. 9 (1968) 1680.

[30] Bonissent, A. and Mutaftschiev, B., Philos. Mag. 35 (1977) 65 


\section{DISCUSSION}

F. A. Putnam. - Recently Binder and Landau [1] have studied the square lattice gas with nearest neighbour repulsion and next nearest neighbour attraction by Monte Carlo methods. Also, Mihura and Landau [2] have made a similar study of the triangular lattice gas. In both of these studies, a line of continuous transitions was found which meets a line of first order transitions at a tricritical point. These studies show that the phase transition phenomena predicted by more sophisticated methods than the Bragg-Williams approximation can be quite different than those predicted by the Bragg-Williams approximation.

[1] BINDER and LANDAU, D. P., Surf. Sci.

[2] Minura, B. and Landau, D. P., Phys. Rev. Lett. 38 (1977)
D. Nicholson. - Are you able to say how the approximations in your calculations (Bragg-Williams and mean field theory) affect your conclusions ?

B. MUTAFTSCHIEV. - Not exactly ; however, the comparison of the Bragg-Williams and the Ising models in the known resolved cases shows that from the Bragg-Williams model one can expect higher critical temperatures, i.e. that this model favors first order transitions in general. On the other hand, we would not expect a first order transition for the ordering of a condensed layer by application of the Ising model to the same (e.g. three to one) case of fixed adsorption. 